\title{
COMMON COUPLED FIXED POINT THEOREMS FOR TWO HYBRID PAIRS OF MAPPINGS SATISFYING AN IMPLICIT RELATION
}

\author{
BHAVANA DESHPANDE AND AMRISH HANDA
}

\begin{abstract}
We establish two common coupled fixed point theorems for two hybrid pairs of mappings satisfying an implicit relation under weak commutativity and $w$-compatibility on a complete metric space, which is not partially ordered. We do not use the condition of continuity of any mapping for finding the coupled coincidence and common coupled fixed point. We improve, extend and generalize several known results.
\end{abstract}

\section{INTRODUCTION AND PRELIMINARIES}

Let $(X, d)$ be a metric space and $C B(X)$ be the set of all nonempty closed bounded subsets of $X$. Let $D(x, A)$ denote the distance from $x$ to $A \subset X$ and $H$ denote the Hausdorff metric induced by $d$, that is,

$$
\begin{aligned}
& D(x, A)=\inf _{a \in A} d(x, a), \\
& H(A, B)=\max \left\{\sup _{a \in A} D(a, B), \sup _{b \in B} D(b, A)\right\}, \text { for all } A, B \in C B(X) .
\end{aligned}
$$

The study of fixed points for multivalued contractions and non-expansive mappings using the Hausdorff metric was studied by many authors under different conditions. The theory of multivalued mappings has found application in control theory, convex optimization, differential inclusions and economics. There exists considerable literature about fixed point properties for two hybrid pairs of mappings, which have been studied by many authors including ([2], [11], [12], [13], [22], [28], [29], [36]).

Bhaskar and Lakshmikantham [8] introduced the concept of coupled fixed point for single-valued mappings and established some coupled fixed point

2010 Mathematics Subject Classification. 47H10, 54H25.

Key words and phrases. Coupled fixed point, coupled coincidence point, implicit relation, $w$-compatibility, weak commuting mappings.

Copyright (C) 2015 by ANUBiH. 
results and found its application in the existence and uniqueness of solution for periodic boundary value problems. Lakshmikantham and Ćirić [20] proved coupled coincidence and common coupled fixed point theorems for nonlinear contractive mappings in partially ordered complete metric spaces and extended the results established in [8]. Many authors focused and proved related remarkable results including ([3], [6], [10], [14], [16], [17], [18], [23], [25], [30], [37]).

Very recently, Samet, Karapinar, Aydi and Rajić [32] claimed that most of the coupled fixed point theorems in the setting of single-valued mappings on ordered metric spaces are consequences of well-known fixed point theorems.

The coupled fixed point theory for multivalued mappings was introduced by Abbas, Ćirić, Damjanović and Khan [1] and obtained coupled coincidence point and common coupled fixed point theorems involving hybrid pair of mappings satisfying generalized contractive conditions in complete metric space.

On the other hand, at present, coupled fixed point theorems for hybrid pair of mappings were studied by very few authors including ([1], [21]).

In [1], Abbas, Ćirić, Damjanović and Khan introduced the following:

Definition 1. Let $X$ be a nonempty set, $F: X \times X \rightarrow 2^{X}$ (a collection of all nonempty subsets of $X)$ and $g$ be a self-mapping on $X$. An element $(x$, $y) \in X \times X$ is called

(1) A coupled fixed point of $F$ if $x \in F(x, y)$ and $y \in F(y, x)$.

(2) A coupled coincidence point of hybrid pair $\{F, g\}$ if $g x \in F(x, y)$ and $g y \in F(y, x)$.

(3) A common coupled fixed point of hybrid pair $\{F, g\}$ if $x=g x \in F(x$, $y)$ and $y=g y \in F(y, x)$.

We denote the set of coupled coincidence points of mappings $F$ and $g$ by $C\{F, g\}$. Note that if $(x, y) \in C\{F, g\}$, then $(y, x)$ is also in $C\{F, g\}$.

Definition 2. Let $F: X \times X \rightarrow 2^{X}$ be a multivalued mapping and $g$ be a self-mapping on $X$. The mapping $g$ is called $F$-weakly commuting at some point $(x, y) \in X \times X$ if $g^{2} x \in F(g x, g y)$ and $g^{2} y \in F(g y, g x)$.

Definition 3. Let $F: X \times X \rightarrow 2^{X}$ be a multivalued mapping and $g$ be a self-mapping on $X$. The hybrid pair $\{F, g\}$ is called $w$-compatible if $g F(x$, $y) \subseteq F(g x, g y)$ whenever $(x, y) \in C\{F, g\}$.

Lemma 1. [15]. Let $(X, d)$ be a metric space. Then, for each $a \in X$ and $B \in C B(X)$, there is $b_{0} \in B$ such that $D(a, B)=d\left(a, b_{0}\right)$, where $D(a$, $B)=\inf _{b \in B} d(a, b)$. 
Fixed point theorems satisfying an implicit relation for single-valued and multivalued mappings under different conditions have been studied by various authors including ([4], [5], [7], [9], [19], [24], [26], [27], [31], [33], [34], [35], [38]).

In this paper, we establish two common coupled fixed point theorems for two hybrid pairs of mappings satisfying an implicit relation under weak commutativity and $w$-compatibility respectively on a complete metric space, which is not partially ordered. We do not use the condition of continuity of any mapping involved therein for finding the coupled coincidence and common coupled fixed point. We improve, extend and generalize the results of Bhaskar and Lakshmikantham [8], Sedghi, Altun and Shobe [33] and many others results in the existing literature.

\section{IMPLICIT RELATION}

Let $R^{+}$be the set of all non-negative real numbers and let $\Psi$ be the set of all continuous functions $\psi:\left(R^{+}\right)^{9} \rightarrow R$ satisfying the following conditions:

$\psi_{1}: \psi\left(t_{1}, t_{2}, \ldots, t_{9}\right)$ is non-decreasing in $t_{1}$ and non-increasing in $t_{2}, t_{3}$, $\ldots, t_{9}$.

$\psi_{2}$ : There exists $0<k<1$ such that for every $u, v, p, q \in R^{+}$such that

$$
\psi(u, v, v, u, u+v, q, q, p, p+q) \leq 0,
$$

or

$$
\psi(u, v, u, v, u+v, q, p, q, p+q) \leq 0,
$$

then $\max \{u, p\} \leq k \max \{v, q\}$.

$\psi_{3}:$ For all $u, v>0$,

$$
\psi(u, u, 0,0,2 u, v, 0,0,2 v)>0 .
$$

Example 1. Let $\psi\left(t_{1}, t_{2}, \ldots, t_{9}\right)=t_{1}-h \max \left\{t_{2}, t_{3}, t_{4}, \frac{t_{5}}{2}, t_{6}, t_{7}, t_{8}, \frac{t_{9}}{2}\right\}$ where $0<h<1$.

$\left(\psi_{1}\right)$ Obvious. $\left(\psi_{2}\right)$ Let $\max \{u, p\}>0$ and $\psi(u, v, v, u, u+v, q, q$, $p, p+q)=u-h \max \{u, v, p, q\} \leq 0$. Thus $u \leq h \max \{\max \{u, p\}$, $\max \{v, q\}\}$. Similarly $p \leq h \max \{\max \{u, p\}, \max \{v, q\}\}$. Thus $\max \{u$, $p\} \leq h \max \{\max \{u, p\}, \max \{v, q\}\}$. Now, if $\max \{u, p\} \geq \max \{v, q\}$, then $\max \{u, p\} \leq h \max \{u, p\}<\max \{u, p\}$, which is a contradiction. Thus $\max \{u, p\}<\max \{v, q\}$ and $\max \{u, p\} \leq h \max \{v, q\}$. Similarly, let $\max \{u$, $p\}>0$ and $\psi(u, v, u, v, u+v, q, p, q, p+q)=u-h \max \{u, v, p, q\} \leq 0$, then we have $\max \{u, p\} \leq h \max \{v, q\}$. Thus $\left(\psi_{2}\right)$ is satisfying with $k=h<1$. If $\max \{u, p\}=0$, then $\max \{u, p\} \leq k \max \{v, q\} .\left(\psi_{3}\right) \psi(u, u, 0,0,2 u, v, 0,0$, $2 v)=u-h \max \{u, v\}=\max \{u-h u, u-h v\}=\max \{u(1-h), u-h v\}>0$.

Therefore $\psi \in \Psi$. 
BHAVANA DESHPANDE AND AMRISH HANDA

Example 2. Let $\psi\left(t_{1}, t_{2}, \ldots, t_{9}\right)=t_{1}-\alpha \max \left\{t_{2}, t_{3}, t_{4}, t_{6}, t_{7}, t_{8}\right\}-$ $\beta \max \left\{t_{5}, t_{9}\right\}$ where $\alpha, \beta \geq 0$ and $\alpha+2 \beta<1$.

$\left(\psi_{1}\right)$ Obvious. $\left(\psi_{2}\right)$ Let $\max \{u, p\}>0$ and $\psi(u, v, v, u, u+v, q, q$, $p, p+q)=u-\alpha \max \{v, u, q, p\}-\beta \max \{u+v, p+q\} \leq 0$, then $u \leq$ $\alpha \max \{\max \{u, p\}, \max \{v, q\}\}+\beta[\max \{u, p\}+\max \{v, q\}]$, it follows that $u \leq \max \{(\alpha+\beta) \max \{u, p\}+\beta \max \{v, q\},(\alpha+\beta) \max \{v, q\}+\beta \max \{u$, $p\}\}$. Similarly $p \leq \max \{(\alpha+\beta) \max \{u, p\}+\beta \max \{v, q\},(\alpha+\beta) \max \{v$, $q\}+\beta \max \{u, p\}\}$. Thus $\max \{u, p\} \leq \max \{(\alpha+\beta) \max \{u, p\}+\beta \max \{v$, $q\},(\alpha+\beta) \max \{v, q\}+\beta \max \{u, p\}\}$. Now, if $\max \{u, p\} \geq \max \{v, q\}$, then $\max \{u, p\} \leq(\alpha+2 \beta) \max \{u, p\}<\max \{u, p\}$, which is a contradiction. Thus $\max \{u, p\}<\max \{v, q\}$ and so $\max \{u, p\} \leq(\alpha+2 \beta) \max \{v, q\}$. Similarly, let $\max \{u, p\}>0$ and $\psi(u, v, u, v, u+v, q, p, q, p+q)=$ $u-\alpha \max \{v, u, q, p\}-\beta \max \{u+v, p+q\} \leq 0$, then we have $\max \{u$, $p\} \leq(\alpha+2 \beta) \max \{v, q\}$. Thus $\left(\psi_{2}\right)$ is satisfying with $k=(\alpha+2 \beta)<1$. If $\max \{u, p\}=0$, then $\max \{u, p\} \leq k \max \{v, q\} .\left(\psi_{3}\right) \psi(u, u, 0,0,2 u, v, 0$, $0,2 v)=u-\alpha \max \{u, v\}-\beta \max \{2 u, 2 v\}=u-\alpha \max \{u, v\}-2 \beta \max \{u$, $v\}=u-(\alpha+2 \beta) \max \{u, v\}=\max \{u(1-\alpha-2 \beta), u-(\alpha+2 \beta) v\}>0$.

Therefore $\psi \in \Psi$.

Example 3. $\psi\left(t_{1}, t_{2}, \ldots, t_{9}\right)=t_{1}-a \max \left\{t_{2}, t_{6}\right\}-b \max \left\{t_{3}+t_{4}, t_{7}+t_{8}\right\}-$ $c \max \left\{t_{5}, t_{9}\right\}$, where $a, b, c \in[0,1)$ and $a+2 b+2 c<1$.

$\left(\psi_{1}\right)$ Obvious. $\left(\psi_{2}\right)$ Let $\max \{u, p\}>0$ and $\psi(u, v, v, u, u+v, q, q, p$, $p+q)=u-a \max \{v, q\}-b \max \{u+v, p+q\}-c \max \{u+v, p+q\} \leq 0$, then $u \leq$ $a \max \{v, q\}+b[\max \{u, p\}+\max \{v, q\}]+c[\max \{u, p\}+\max \{v, q\}]$. Similarly $p \leq a \max \{v, q\}+b[\max \{u, p\}+\max \{v, q\}]+c[\max \{u, p\}+\max \{v, q\}]$. Thus $\max \{u, p\} \leq a \max \{v, q\}+b[\max \{u, p\}+\max \{v, q\}]+c[\max \{u, p\}+\max \{v$, $q\}]$. Now, if $\max \{u, p\} \geq \max \{v, q\}$, then $\max \{u, p\} \leq(a+2 b+2 c) \max \{u$, $p\}<\max \{u, p\}$, which is a contradiction. Thus $\max \{u, p\}<\max \{v, q\}$ and $\max \{u, p\} \leq(a+2 b+2 c) \max \{v, q\}$. Similarly, let $\max \{u, p\}>0$ and $\psi(u, v, u, v, u+v, q, p, q, p+q)=u-a \max \{v, q\}-b \max \{u+v$, $p+q\}-c \max \{u+v, p+q\} \leq 0$, then $\max \{u, p\}<(a+2 b+2 c) \max \{v$, $q$. Thus $\left(\psi_{2}\right)$ is satisfying with $k=a+2 b+2 c<1$. If $\max \{u, p\}=0$, then $\max \{u, p\} \leq k \max \{v, q\} .\left(\psi_{3}\right) \psi(u, u, 0,0,2 u, v, 0,0,2 v)=u-a \max \{u$, $v\}-c \max \{2 u, 2 v\}=u-a \max \{u, v\}-2 c \max \{u, v\}=u-(a+2 c) \max \{u$, $v\}=\max \{u(1-a-2 c), u-(a+2 c) v\}>0$.

Therefore $\psi \in \Psi$.

\section{MAin RESUlts}

Theorem 1. Let $(X, d)$ be a complete metric space. Assume $F, G: X \times X \rightarrow$ $C B(X)$ and $f, g: X \rightarrow X$ be mappings satisfying

(1.1) $F(X \times X) \subseteq g(X), G(X \times X) \subseteq f(X)$, 
(1.2) for all $x, y, u, v \in X$ and $\psi \in \Psi$,

$$
\psi\left(\begin{array}{c}
H(F(x, y), G(u, v)), \\
d(f x, g u), D(f x, F(x, y)), D(g u, G(u, v)), \\
D(f x, G(u, v))+D(g u, F(x, y)), \\
d(f y, g v), D(f y, F(y, x)), D(g v, G(v, u)), \\
D(f y, G(v, u))+D(g v, F(y, x))
\end{array}\right) \leq 0
$$

(1.3) $f(X)$ and $g(X)$ are closed subsets of $X$, then

(a) $F$ and $f$ have a coupled coincidence point,

(b) $G$ and $g$ have a coupled coincidence point,

(c) $F$ and $f$ have a common coupled fixed point, if $f$ is $F$-weakly commuting at $(x, y)$ and $f^{2} x=f x$ and $f^{2} y=f y$ for $(x, y) \in C\{F, f\}$,

(d) $G$ and $g$ have a common coupled fixed point, if $g$ is $G$-weakly commuting at $(\widetilde{x}, \widetilde{y})$ and $g^{2} \widetilde{x}=g \widetilde{x}$ and $g^{2} \widetilde{y}=g \widetilde{y}$ for $(\widetilde{x}, \widetilde{y}) \in C\{G, g\}$,

(e) $F, G, f, g$ have common coupled fixed point provided that both (c) and (d) are true.

Proof. Let $x_{0}, y_{0} \in X$ be arbitrary. Choose $u_{1}=g x_{1} \in F\left(x_{0}, y_{0}\right)$ and $v_{1}=g y_{1} \in F\left(y_{0}, x_{0}\right)$, as $F(X \times X) \subseteq g(X)$. Since $F, G: X \times X \rightarrow C B(X)$, therefore by Lemma 1 , there exist $u_{2} \in G\left(x_{1}, y_{1}\right)$ and $v_{2} \in G\left(y_{1}, x_{1}\right)$ such that

$$
\begin{aligned}
d\left(u_{1}, u_{2}\right) & \leq H\left(F\left(x_{0}, y_{0}\right), G\left(x_{1}, y_{1}\right)\right), \\
d\left(v_{1}, v_{2}\right) & \leq H\left(F\left(y_{0}, x_{0}\right), G\left(y_{1}, x_{1}\right)\right) .
\end{aligned}
$$

Since $G(X \times X) \subseteq f(X)$, there exist $x_{2}, y_{2} \in X$ such that $u_{2}=f x_{2} \in G\left(x_{1}\right.$, $\left.y_{1}\right)$ and $v_{2}=f y_{2} \in G\left(y_{1}, x_{1}\right)$. Then we choose $u_{3} \in F\left(x_{2}, y_{2}\right)$ and $v_{3} \in F\left(y_{2}\right.$, $x_{2}$ ) such that

$$
\begin{aligned}
d\left(u_{2}, u_{3}\right) & \leq H\left(G\left(x_{1}, y_{1}\right), F\left(x_{2}, y_{2}\right)\right), \\
d\left(v_{2}, v_{3}\right) & \leq H\left(G\left(y_{1}, x_{1}\right), F\left(y_{2}, x_{2}\right)\right) .
\end{aligned}
$$

Continuing this process, we obtain sequences $\left\{u_{n}\right\},\left\{v_{n}\right\},\left\{x_{n}\right\}$ and $\left\{y_{n}\right\}$ in $X$ such that for all $n \geq 0$, we have

$$
\begin{aligned}
& u_{2 n}=f x_{2 n} \in G\left(x_{2 n-1}, y_{2 n-1}\right), u_{2 n+1}=g x_{2 n+1} \in F\left(x_{2 n}, y_{2 n}\right), \\
& v_{2 n}=f y_{2 n} \in G\left(y_{2 n-1}, x_{2 n-1}\right), v_{2 n+1}=g y_{2 n+1} \in F\left(y_{2 n}, x_{2 n}\right),
\end{aligned}
$$

and

$$
\begin{aligned}
d\left(u_{2 n-1}, u_{2 n}\right) & \leq H\left(F\left(x_{2 n-2}, y_{2 n-2}\right), G\left(x_{2 n-1}, y_{2 n-1}\right)\right), \\
d\left(u_{2 n}, u_{2 n+1}\right) & \leq H\left(G\left(x_{2 n-1}, y_{2 n-1}\right), F\left(x_{2 n}, y_{2 n}\right)\right), \\
d\left(v_{2 n-1}, v_{2 n}\right) & \leq H\left(F\left(y_{2 n-2}, x_{2 n-2}\right), G\left(y_{2 n-1}, x_{2 n-1}\right)\right), \\
d\left(v_{2 n}, v_{2 n+1}\right) & \leq H\left(G\left(y_{2 n-1}, x_{2 n-1}\right), F\left(y_{2 n}, x_{2 n}\right)\right) .
\end{aligned}
$$


Then by condition (1.2), we get

$$
\psi\left(\begin{array}{c}
H\left(F\left(x_{2 n}, y_{2 n}\right), G\left(x_{2 n-1}, y_{2 n-1}\right)\right), \\
d\left(f x_{2 n}, g x_{2 n-1}\right), D\left(f x_{2 n}, F\left(x_{2 n}, y_{2 n}\right)\right), \\
D\left(g x_{2 n-1}, G\left(x_{2 n-1}, y_{2 n-1}\right)\right), \\
D\left(f x_{2 n}, G\left(x_{2 n-1}, y_{2 n-1}\right)+D\left(g x_{2 n-1}, F\left(x_{2 n}, y_{2 n}\right)\right),\right. \\
d\left(f y_{2 n}, g y_{2 n-1}\right), D\left(f y_{2 n}, F\left(y_{2 n}, x_{2 n}\right)\right), \\
D\left(g y_{2 n-1}, G\left(y_{2 n-1}, x_{2 n-1}\right)\right), \\
D\left(f y_{2 n}, G\left(y_{2 n-1}, x_{2 n-1}\right)\right)+D\left(g y_{2 n-1}, F\left(y_{2 n}, x_{2 n}\right)\right)
\end{array}\right) \leq 0 .
$$

Using $\left(\psi_{1}\right)$, we get

$$
\psi\left(\begin{array}{c}
d\left(u_{2 n+1}, u_{2 n}\right), \\
d\left(u_{2 n}, u_{2 n-1}\right), d\left(u_{2 n}, u_{2 n+1}\right), d\left(u_{2 n-1}, u_{2 n}\right), \\
0+d\left(u_{2 n-1}, u_{2 n+1}\right) \\
d\left(v_{2 n}, v_{2 n-1}\right), d\left(v_{2 n}, v_{2 n+1}\right), d\left(v_{2 n-1}, v_{2 n}\right), \\
0+d\left(v_{2 n-1}, v_{2 n+1}\right)
\end{array}\right) \leq 0
$$

which implies that

$$
\psi\left(\begin{array}{c}
d\left(u_{2 n+1}, u_{2 n}\right), \\
d\left(u_{2 n}, u_{2 n-1}\right), d\left(u_{2 n}, u_{2 n+1}\right), d\left(u_{2 n-1}, u_{2 n}\right), \\
d\left(u_{2 n-1}, u_{2 n}\right)+d\left(u_{2 n}, u_{2 n+1}\right), \\
d\left(v_{2 n}, v_{2 n-1}\right), d\left(v_{2 n}, v_{2 n+1}\right), d\left(v_{2 n-1}, v_{2 n}\right), \\
d\left(v_{2 n-1}, v_{2 n}\right)+d\left(v_{2 n}, v_{2 n+1}\right)
\end{array}\right) \leq 0
$$

By $\left(\psi_{2}\right)$, we get

$$
\begin{aligned}
\max \left\{d\left(u_{2 n+1}, u_{2 n}\right), d\left(v_{2 n+1},\right.\right. & \left.\left.v_{2 n}\right)\right\} \\
& \leq k \max \left\{d\left(u_{2 n}, u_{2 n-1}\right), d\left(v_{2 n}, v_{2 n-1}\right)\right\} .
\end{aligned}
$$

Similarly, we can obtain

$$
\begin{aligned}
& \max \left\{d\left(u_{2 n}, u_{2 n-1}\right), d\left(v_{2 n}, v_{2 n-1}\right)\right\} \\
& \quad \leq k \max \left\{d\left(u_{2 n-1}, u_{2 n-2}\right), d\left(v_{2 n-1}, v_{2 n-2}\right)\right\} .
\end{aligned}
$$

Thus, we have for all $n \in \mathbb{N}$,

$$
\begin{aligned}
\max \left\{d\left(u_{n}, u_{n+1}\right), d\left(v_{n}, v_{n+1}\right)\right\} & \leq k \max \left\{d\left(u_{n-1}, u_{n}\right), d\left(v_{n-1}, v_{n}\right)\right\} \\
& \leq k^{n} \max \left\{d\left(u_{0}, u_{1}\right), d\left(v_{0}, v_{1}\right)\right\} \\
& \leq k^{n} \delta
\end{aligned}
$$

Thus

$$
\max \left\{d\left(u_{n}, u_{n+1}\right), d\left(v_{n}, v_{n+1}\right)\right\} \leq k^{n} \delta,
$$

where

$$
\delta=\max \left\{d\left(u_{0}, u_{1}\right), d\left(v_{0}, v_{1}\right)\right\}
$$


Thus, for $m, n \in \mathbb{N}$ with $m>n$, by triangle inequality and (1.4), we get

$$
\begin{aligned}
& \max \left\{d\left(u_{n}, u_{m+n}\right), d\left(v_{n}, v_{m+n}\right)\right\} \leq \max \left\{d\left(u_{n}, u_{n+1}\right), d\left(v_{n}, v_{n+1}\right)\right\} \\
& +\max \left\{d\left(u_{n+1}, u_{n+2}\right), d\left(v_{n+1}, v_{n+2}\right)\right\} \\
& \quad+\cdots+\max \left\{d\left(u_{m+n-1}, u_{m+n}\right), d\left(v_{m+n-1}, v_{m+n}\right)\right\} \\
& \leq k^{n} \delta+k^{n+1} \delta+\cdots+k^{n+m-1} \delta \\
& \leq \sum_{i=n}^{n+m-1} k^{i} \delta
\end{aligned}
$$

which shows that $\left\{u_{n}\right\}$ and $\left\{v_{n}\right\}$ are Cauchy sequences in $X$. Since $X$ is complete, there exist $u, v \in X$ such that

$$
\begin{aligned}
& \lim _{n \rightarrow \infty} u_{n}=\lim _{n \rightarrow \infty} f x_{2 n}=\lim _{n \rightarrow \infty} g x_{2 n+1}=u, \\
& \lim _{n \rightarrow \infty} v_{n}=\lim _{n \rightarrow \infty} f y_{2 n}=\lim _{n \rightarrow \infty} g y_{2 n+1}=v .
\end{aligned}
$$

Since $f(X)$ and $g(X)$ are closed subsets of $X$, then there exist $x, y, \widetilde{x}, \widetilde{y} \in X$,

$$
u=f x=g \widetilde{x} \text { and } v=f y=g \widetilde{y} .
$$

Now, since $f x_{2 n} \in G\left(x_{2 n-1}, y_{2 n-1}\right)$ and $f y_{2 n} \in G\left(y_{2 n-1}, x_{2 n-1}\right)$, therefore by using condition (1.2), we get

$$
\psi\left(\begin{array}{c}
H\left(F(x, y), G\left(x_{2 n-1}, y_{2 n-1}\right)\right) \\
d\left(f x, g x_{2 n-1}\right), D(f x, F(x, y)) D\left(g x_{2 n-1}, G\left(x_{2 n-1}, y_{2 n-1}\right)\right), \\
D\left(f x, G\left(x_{2 n-1}, y_{2 n-1}\right)\right)+D\left(g x_{2 n-1}, F(x, y)\right), \\
d\left(f y, g y_{2 n-1}\right), D(f y, F(y, x)) D\left(g y_{2 n-1}, G\left(y_{2 n-1}, x_{2 n-1}\right)\right), \\
D\left(f y, G\left(y_{2 n-1}, x_{2 n-1}\right)\right)+D\left(g y_{2 n-1}, F(y, x)\right)
\end{array}\right) \leq 0
$$

which implies, by $\left(\psi_{1}\right)$, that

$$
\psi\left(\begin{array}{c}
D\left(F(x, y), f x_{2 n}\right), \\
d\left(f x, g x_{2 n-1}\right), D(f x, F(x, y)), d\left(g x_{2 n-1}, f x_{2 n}\right), \\
d\left(f x, f x_{2 n}\right)+D\left(g x_{2 n-1}, F(x, y)\right), \\
d\left(f y, g y_{2 n-1}\right), D(f y, F(y, x)), d\left(g y_{2 n-1}, f y_{2 n}\right), \\
d\left(f y, f y_{2 n}\right)+D\left(g y_{2 n-1}, F(y, x)\right)
\end{array}\right) \leq 0
$$

Letting $n \rightarrow \infty$ in the above inequality, by using the continuity of $\psi,(1.5)$ and (1.6), we obtain

$$
\psi\left(\begin{array}{c}
D(F(x, y), f x), \\
0, D(f x, F(x, y)), 0,0+D(f x, F(x, y)), \\
0, D(f y, F(y, x)), 0,0+D(f y, F(y, x))
\end{array}\right) \leq 0 .
$$

Thus, by $\left(\psi_{2}\right)$, we obtain

$$
D(f x, F(x, y))=0 \text { and } D(f y, F(y, x))=0,
$$


which implies that

$$
f x \in F(x, y) \text { and } f y \in F(y, x),
$$

that is, $(x, y)$ is a coupled coincidence point of $F$ and $f$. This proves $(a)$. Again, since $g x_{2 n+1} \in F\left(x_{2 n}, y_{2 n}\right)$ and $g y_{2 n+1} \in F\left(y_{2 n}, x_{2 n}\right)$, therefore by using condition (1.2), we get

$$
\psi\left(\begin{array}{c}
H\left(F\left(x_{2 n}, y_{2 n}\right), G(\widetilde{x}, \widetilde{y})\right), \\
d\left(f x_{2 n}, g \widetilde{x}\right), D\left(f x_{2 n}, F\left(x_{2 n}, y_{2 n}\right)\right), D(g \widetilde{x}, G(\widetilde{x}, \widetilde{y})), \\
D\left(f x_{2 n}, G(\widetilde{x}, \widetilde{y})\right)+D\left(g \widetilde{x}, F\left(x_{2 n}, y_{2 n}\right)\right), \\
d\left(f y_{2 n}, g \widetilde{y}\right), D\left(f y_{2 n}, F\left(y_{2 n}, x_{2 n}\right)\right), D(g \widetilde{y}, G(\widetilde{y}, \widetilde{x})), \\
D\left(f y_{2 n}, G(\widetilde{y}, \widetilde{x})\right)+D\left(g \widetilde{y}, F\left(y_{2 n}, x_{2 n}\right)\right)
\end{array}\right) \leq 0,
$$

which implies, by $\left(\psi_{1}\right)$, that

$$
\psi\left(\begin{array}{c}
D\left(g x_{2 n+1}, G(\widetilde{x}, \widetilde{y})\right) \\
d\left(f x_{2 n}, g \widetilde{x}\right), d\left(f x_{2 n}, g x_{2 n+1}\right), D(g \widetilde{x}, G(\widetilde{x}, \widetilde{y})), \\
D\left(f x_{2 n}, G(\widetilde{x}, \widetilde{y})\right)+d\left(g \widetilde{x}, g x_{2 n+1}\right), \\
d\left(f y_{2 n}, g \widetilde{y}\right), d\left(f y_{2 n}, g y_{2 n+1}\right), D(g \widetilde{y}, G(\widetilde{y}, \widetilde{x})), \\
D\left(f y_{2 n}, G(\widetilde{y}, \widetilde{x})\right)+d\left(g \widetilde{y}, g y_{2 n+1}\right)
\end{array}\right) \leq 0
$$

Letting $n \rightarrow \infty$ in the above inequality, by using the continuity of $\psi,(1.5)$ and (1.6), we obtain

$$
\psi\left(\begin{array}{c}
D(g \widetilde{x}, G(\widetilde{x}, \widetilde{y})) \\
0,0, D(g \widetilde{x}, G(\widetilde{x}, \widetilde{y})), D(g \widetilde{x}, G(\widetilde{x}, \widetilde{y}))+0 \\
0,0, D(g \widetilde{y}, G(\widetilde{y}, \widetilde{x})), D(g \widetilde{y}, G(\widetilde{y}, \widetilde{x}))+0
\end{array}\right) \leq 0 .
$$

Thus, by $\left(\psi_{2}\right)$, we obtain

$$
D(g \widetilde{x}, G(\widetilde{x}, \widetilde{y}))=0 \text { and } D(g \widetilde{y}, G(\widetilde{y}, \widetilde{x}))=0,
$$

which implies that

$$
g \widetilde{x} \in G(\widetilde{x}, \widetilde{y}) \text { and } g \widetilde{y} \in G(\widetilde{y}, \widetilde{x}) .
$$

that is, $(\widetilde{x}, \widetilde{y})$ is a coupled coincidence point of $G$ and $g$. This proves $(b)$.

Furthermore, from condition $(c)$, we have $f$ is $F$-weakly commuting at ( $x$, $y)$, that is, $f^{2} x \in F(f x, f y), f^{2} y \in F(f y, f x)$ and $f^{2} x=f x, f^{2} y=f y$. Thus $f x=f^{2} x \in F(f x, f y)$ and $f y=f^{2} y \in F(f y, f x)$, that is, $u=f u \in F(u$, $v$ ) and $v=f v \in F(v, u)$. This proves $(c)$. A similar argument proves $(d)$. Then $(e)$ holds immediately.

Put $f=g$ in the Theorem 1, we get the following result:

Corollary 2. Let $(X, d)$ be a complete metric space. Assume $F, G: X \times$ $X \rightarrow C B(X)$ and $g: X \rightarrow X$ be mappings satisfying

(2.1) $F(X \times X) \subseteq g(X), G(X \times X) \subseteq g(X)$, 
(2.2) for all $x, y, u, v \in X$ and $\psi \in \Psi$,

$$
\psi\left(\begin{array}{c}
H(F(x, y), G(u, v)), \\
d(g x, g u), D(g x, F(x, y)), D(g u, G(u, v)), \\
D(g x, G(u, v))+D(g u, F(x, y)), \\
d(g y, g v), D(g y, F(y, x)), D(g v, G(v, u)), \\
D(g y, G(v, u))+D(g v, F(y, x))
\end{array}\right) \leq 0 .
$$

(2.3) $g(X)$ is a closed subset of $X$, then

(a) $F$ and $g$ have a coupled coincidence point,

(b) $G$ and $g$ have a coupled coincidence point,

(c) $F$ and $g$ have a common coupled fixed point, if $g$ is $F$-weakly commuting at $(x, y)$ and $g^{2} x=g x$ and $g^{2} y=g y$ for $(x, y) \in C\{F, g\}$,

(d) $G$ and $g$ have a common coupled fixed point, if $g$ is $G$-weakly commuting at $(\widetilde{x}, \widetilde{y})$ and $g^{2} \widetilde{x}=g \widetilde{x}$ and $g^{2} \widetilde{y}=g \widetilde{y}$ for $(\widetilde{x}, \widetilde{y}) \in C\{G, g\}$,

(e) $F, G, g$ have common coupled fixed point provided that both (c) and (d) are true.

Put $F=G$ and $f=g$ in the Theorem 1, we get the following result:

Corollary 3. Let $(X, d)$ be a complete metric space. Assume $F: X \times X \rightarrow$ $C B(X)$ and $g: X \rightarrow X$ be mappings satisfying

(3.1) $F(X \times X) \subseteq g(X)$

(3.2) for all $x, y, u, v \in X$ and $\psi \in \Psi$,

$$
\psi\left(\begin{array}{c}
H(F(x, y), F(u, v)), \\
d(g x, g u), D(g x, F(x, y)), D(g u, F(u, v)), \\
D(g x, F(u, v))+D(g u, F(x, y)), \\
d(g y, g v), D(g y, F(y, x)), D(g v, F(v, u)), \\
D(g y, F(v, u))+D(g v, F(y, x))
\end{array}\right) \leq 0 .
$$

If (2.3) holds, then

(a) $F$ and $g$ have a coupled coincidence point,

(b) $F$ and $g$ have a common coupled fixed point, if $g$ is $F$-weakly commuting at $(x, y)$ and $g^{2} x=g x$ and $g^{2} y=g y$ for $(x, y) \in C\{F, g\}$.

Examples 1-3 and Theorem 1 imply the following:

Corollary 4. Let $(X, d)$ be a complete metric space. Assume $F, G: X \times$ $X \rightarrow C B(X)$ and $f, g: X \rightarrow X$ be mappings satisfying (1.1) and

(4.1) for all $x, y, u, v \in X$, where $0<h<1$,

$$
\begin{aligned}
& H(F(x, y), G(u, v)) \\
\leq & h \max \left\{\begin{array}{c}
d(f x, g u), D(f x, F(x, y)), D(g u, G(u, v)), \\
d(f y, g v), D(f y, F(y, x)), D(g v, G(v, u)), \\
\frac{D(f x, G(u, v))+D(g u, F(x, y))}{2}, \frac{D(f y, G(v, u))+D(g v, F(y, x))}{2}
\end{array}\right\},
\end{aligned}
$$


or for all $x, y, u, v \in X$, where $\alpha, \beta \geq 0$ and $\alpha+2 \beta<1$,

$$
\begin{aligned}
& H(F(x, y), G(u, v)) \\
& \leq \alpha \max \left\{\begin{array}{c}
d(f x, g u), D(f x, F(x, y)), D(g u, G(u, v)), \\
d(f y, g v), D(f y, F(y, x)), D(g v, G(v, u))
\end{array}\right\} \\
& +\beta \max \left\{\begin{array}{c}
D(f x, G(u, v))+D(g u, F(x, y)), \\
D(f y, G(v, u))+D(g v, F(y, x))
\end{array}\right\},
\end{aligned}
$$

or for all $x, y, u, v \in X$, where $a, b, c \in[0,1)$ and $a+2 b+2 c<1$,

$$
\begin{aligned}
& H(F(x, y), G(u, v)) \\
& \leq a \max \{d(f x, g u), d(f y, g v)\} \\
& \quad+b \max \left\{\begin{array}{c}
D(f x, F(x, y))+D(g u, G(u, v)), \\
D(f y, F(y, x))+D(g v, G(v, u))
\end{array}\right\} \\
& \quad+c \max \left\{\begin{array}{cc}
D(f x, & G(u, v))+D(g u, F(x, y)), \\
D(f y, G(v, u))+D(g v, F(y, x))
\end{array}\right\} .
\end{aligned}
$$

If (1.3) holds, then

(a) $F$ and $f$ have a coupled coincidence point,

(b) $G$ and $g$ have a coupled coincidence point,

(c) $F$ and $f$ have a common coupled fixed point, if $f$ is $F$-weakly commuting at $(x, y)$ and $f^{2} x=f x$ and $f^{2} y=f y$ for $(x, y) \in C\{F, f\}$,

(d) $G$ and $g$ have a common coupled fixed point, if $g$ is $G$-weakly commuting at $(\widetilde{x}, \widetilde{y})$ and $g^{2} \widetilde{x}=g \widetilde{x}$ and $g^{2} \widetilde{y}=g \widetilde{y}$ for $(\widetilde{x}, \widetilde{y}) \in C\{G, g\}$,

(e) $F, G, f, g$ have common coupled fixed point provided that both (c) and (d) are true.

Examples 1-3 and Corollary 2 imply the following:

Corollary 5. Let $(X, d)$ be a complete metric space. Assume $F, G: X \times$ $X \rightarrow C B(X)$ and $g: X \rightarrow X$ be mappings satisfying (2.1) and

(5.1) for all $x, y, u, v \in X$, where $0<h<1$,

$$
\begin{aligned}
& H(F(x, y), G(u, v)) \\
\leq & h \max \left\{\begin{array}{c}
d(g x, g u), D(g x, F(x, y)), D(g u, G(u, v)), \\
d(g y, g v), D(g y, F(y, x)), D(g v, G(v, u)), \\
\frac{D(g x, G(u, v))+D(g u, F(x, y))}{2}, \frac{D(g y, G(v, u))+D(g v, F(y, x))}{2}
\end{array}\right\},
\end{aligned}
$$

or for all $x, y, u, v \in X$, where $\alpha, \beta \geq 0$ and $\alpha+2 \beta<1$,

$$
\begin{aligned}
& H(F(x, y), G(u, v)) \\
\leq & \alpha \max \left\{\begin{array}{c}
d(g x, g u), D(g x, F(x, y)), D(g u, G(u, v)), \\
d(g y, g v), D(g y, F(y, x)), D(g v, G(v, u))
\end{array}\right\} \\
& +\beta \max \left\{\begin{array}{c}
D(g x, G(u, v))+D(g u, F(x, y)), \\
D(g y, G(v, u))+D(g v, F(y, x))
\end{array}\right\},
\end{aligned}
$$


or for all $x, y, u, v \in X$, where $a, b, c \in[0,1)$ and $a+2 b+2 c<1$,

$$
\begin{aligned}
& H(F(x, y), G(u, v)) \\
\leq & a \max \{d(g x, g u), d(g y, g v)\} \\
& +b \max \left\{\begin{array}{l}
D(g x, F(x, y))+D(g u, G(u, v)), \\
D(g y, F(y, x))+D(g v, G(v, u))
\end{array}\right\} \\
& +c \max \left\{\begin{array}{cc}
D(g x, & G(u, v))+D(g u, F(x, y)), \\
D(g y, & G(v, u))+D(g v, F(y, x))
\end{array}\right\} .
\end{aligned}
$$

If (2.3) holds, then

(a) $F$ and $g$ have a coupled coincidence point,

(b) $G$ and $g$ have a coupled coincidence point,

(c) $F$ and $g$ have a common coupled fixed point, if $g$ is $F$-weakly commuting at $(x, y)$ and $g^{2} x=g x$ and $g^{2} y=g y$ for $(x, y) \in C\{F, g\}$,

(d) $G$ and $g$ have a common coupled fixed point, if $g$ is $G$-weakly commuting at $(\widetilde{x}, \widetilde{y})$ and $g^{2} \widetilde{x}=g \widetilde{x}$ and $g^{2} \widetilde{y}=g \widetilde{y}$ for $(\widetilde{x}, \widetilde{y}) \in C\{G, g\}$,

(e) $F, G, g$ have common coupled fixed point provided that both (c) and (d) are true.

Examples 1-3 and Corollary 3 imply the following:

Corollary 6. Let $(X, d)$ be a complete metric space. Assume $F: X \times X \rightarrow$ $C B(X)$ and $g: X \rightarrow X$ be mappings satisfying (3.1) and

(6.1) for all $x, y, u, v \in X$, where $0<h<1$,

$$
\begin{aligned}
& H(F(x, y), F(u, v)) \\
\leq & h \max \left\{\begin{array}{c}
d(g x, g u), D(g x, F(x, y)), D(g u, F(u, v)), \\
d(g y, g v), D(g y, F(y, x)), D(g v, F(v, u)), \\
\frac{D(g x, F(u, v))+D(g u, F(x, y))}{2}, \frac{D(g y, F(v, u))+D(g v, F(y, x))}{2}
\end{array}\right\},
\end{aligned}
$$

or for all $x, y, u, v \in X$, where $\alpha, \beta \geq 0$ and $\alpha+2 \beta<1$,

$$
\begin{aligned}
& H(F(x, y), F(u, v)) \\
\leq & \alpha \max \left\{\begin{array}{c}
d(g x, g u), D(g x, F(x, y)), D(g u, F(u, v)), \\
d(g y, g v), D(g y, F(y, x)), D(g v, F(v, u))
\end{array}\right\} \\
+ & \beta \max \left\{\begin{array}{c}
D(g x, F(u, v))+D(g u, F(x, y)), \\
D(g y, F(v, u))+D(g v, F(y, x))
\end{array}\right\},
\end{aligned}
$$

or for all $x, y, u, v \in X$, where $a, b, c \in[0,1)$ and $a+2 b+2 c<1$,

$$
\begin{aligned}
& H(F(x, y), F(u, v)) \\
\leq & a \max \{d(g x, g u), d(g y, g v)\} \\
& +b \max \left\{\begin{array}{c}
D(g x, F(x, y))+D(g u, F(u, v)), \\
D(g y, F(y, x))+D(g v, F(v, u))
\end{array}\right\}
\end{aligned}
$$




$$
+c \max \left\{\begin{array}{c}
D(g x, F(u, v))+D(g u, F(x, y)) \\
D(g y, F(v, u))+D(g v, F(y, x))
\end{array}\right\} .
$$

If (2.3) holds, then

(a) $F$ and $g$ have a coupled coincidence point,

(c) $F$ and $g$ have a common coupled fixed point, if $g$ is $F$-weakly commuting at $(x, y)$ and $g^{2} x=g x$ and $g^{2} y=g y$ for $(x, y) \in C\{F, g\}$.

Theorem 7. Let $(X, d)$ be a complete metric space. Assume $F, G: X \times X \rightarrow$ $C B(X)$ and $f, g: X \rightarrow X$ be mappings satisfying (1.1), (1.2) and

(7.1) $\{F, f\}$ and $\{G, g\}$ are $w$-compatible,

(7.2) $f(X)$ or $g(X)$ is a closed subset of $X$,

then $F, G, f, g$ have a common coupled fixed point.

Proof. We can prove like Theorem 1 that $\left\{u_{n}\right\}$ and $\left\{v_{n}\right\}$ are Cauchy sequences in $X$. Since $X$ is complete, there exist $u, v \in X$ satisfying (1.5). Suppose that $f(X)$ is a closed subset of $X$, then there exist $x, y \in X$, we have

$$
u=f x \text { and } v=f y .
$$

As in Theorem 1, we can prove that

$$
f x \in F(x, y) \text { and } f y \in F(y, x),
$$

that is, $(x, y)$ is a coupled coincidence point of $F$ and $f$. Hence $(x, y) \in C\{F$, $f\}$. From $w$-compatibility of $\{F, f\}$, we have $f F(x, y) \subseteq F(f x, f y)$, hence $f^{2} x \in F(f x, f y)$ and $f^{2} y \in F(f y, f x)$, that is, $f u \in F(u, v)$ and $f v \in F(v$, $u)$. Now, by condition (1.2), we get

$$
\psi\left(\begin{array}{c}
H\left(F(u, v), G\left(x_{2 n-1}, y_{2 n-1}\right)\right), \\
d\left(f u, g x_{2 n-1}\right), D(f u, F(u, v)), D\left(g x_{2 n-1}, G\left(x_{2 n-1}, y_{2 n-1}\right)\right), \\
D\left(f u, G\left(x_{2 n-1}, y_{2 n-1}\right)\right)+D\left(g x_{2 n-1}, F(u, v)\right), \\
d\left(f v, g y_{2 n-1}\right), D(f v, F(v, u)), D\left(g y_{2 n-1}, G\left(y_{2 n-1}, x_{2 n-1}\right)\right), \\
D\left(f v, G\left(y_{2 n-1}, x_{2 n-1}\right)\right)+D\left(g y_{2 n-1}, F(v, u)\right)
\end{array}\right) \leq 0 .
$$

From $\left(\psi_{1}\right)$ and by triangle inequality, we have

$$
\psi\left(\begin{array}{c}
d\left(f u, u_{2 n}\right), \\
d\left(f u, u_{2 n-1}\right), 0, d\left(u_{2 n-1}, u_{2 n}\right), d\left(f u, u_{2 n}\right)+d\left(u_{2 n-1}, f u\right), \\
d\left(f v, v_{2 n-1}\right), 0, d\left(v_{2 n-1}, v_{2 n}\right), d\left(f v, v_{2 n}\right)+d\left(v_{2 n-1}, f v\right)
\end{array}\right) \leq 0 .
$$

Letting $n \rightarrow \infty$ in the above inequality, we get

$$
\psi\left(\begin{array}{c}
d(f u, u) \\
d(f u, u), 0,0,2 d(f u, u), \\
d(f v, v), 0,0,2 d(f v, v)
\end{array}\right) \leq 0
$$

Hence, by $\left(\psi_{3}\right)$, we have $d(f u, u)=d(f v, v)=0$. Thus

$$
u=f u \in F(u, v) \text { and } v=f v \in F(v, u) .
$$


Since $F(X \times X) \subseteq g(X)$, then there exist $\widetilde{x}, \widetilde{y} \in X$ such that $g \widetilde{x}=u=$ $f u \in F(u, v)$ and $g \widetilde{y}=v=f v \in F(v, u)$. Again, by condition (1.2), we get

$$
\psi\left(\begin{array}{c}
H(F(u, v), G(\widetilde{x}, \widetilde{y})), \\
d(f u, g \widetilde{x}), D(f u, F(u, v)), D(g \widetilde{x}, G(\widetilde{x}, \widetilde{y})), \\
D(f u, G(\widetilde{x}, \widetilde{y}))+D(g \widetilde{x}, F(u, v)), \\
d(f v, g \widetilde{y}), D(f v, F(v, u)), D(g \widetilde{y}, G(\widetilde{y}, \widetilde{x})), \\
D(f v, G(\widetilde{y}, \widetilde{x}))+D(g \widetilde{y}, F(v, u))
\end{array}\right) \leq 0 .
$$

From $\left(\psi_{1}\right)$, we have

$$
\psi\left(\begin{array}{c}
D(u, G(\widetilde{x}, \widetilde{y})) \\
0,0, D(u, G(\widetilde{x}, \widetilde{y})), D(u, G(\widetilde{x}, \widetilde{y}))+0 \\
0,0, D(v, G(\widetilde{y}, \widetilde{x})), D(v, G(\widetilde{y}, \widetilde{x}))+0
\end{array}\right) \leq 0 .
$$

Hence, by $\left(\psi_{2}\right)$, we have $D(u, G(\widetilde{x}, \widetilde{y}))=D(v, G(\widetilde{y}, \widetilde{x}))=0$. Thus

$$
u=g \widetilde{x} \in G(\widetilde{x}, \widetilde{y}) \text { and } v=g \widetilde{y} \in G(\widetilde{y}, \widetilde{x}),
$$

that is, $(\widetilde{x}, \widetilde{y})$ is a coupled coincidence point of $G$ and $g$. Hence $(\widetilde{x}, \widetilde{y}) \in C\{G$, $g\}$. From $w$-compatibility of $\{G, g\}$, we have $g G(\widetilde{x}, \widetilde{y}) \subseteq G(g \widetilde{x}, g \widetilde{y})$, hence $g^{2} \widetilde{x} \in G(g \widetilde{x}, g \widetilde{y})$ and $g^{2} \widetilde{y} \in G(g \widetilde{y}, g \widetilde{x})$, that is, $g u \in G(u, v)$ and $g v \in G(v$, $u$ ). Now, by condition (1.2), we get

$$
\psi\left(\begin{array}{c}
H(F(u, v), G(u, v)), \\
d(f u, g u), D(f u, F(u, v)), D(g u, G(u, v)), \\
D(f u, G(u, v))+D(g u, F(u, v)), \\
d(f v, g v), D(f v, F(v, u)), D(g v, G(v, u)), \\
D(f v, G(v, u))+D(g v, F(v, u))
\end{array}\right) \leq 0 .
$$

From $\left(\psi_{2}\right)$ and triangle inequality, we have

$$
\psi\left(\begin{array}{c}
d(u, g u) \\
d(u, g u), 0,0,2 d(u, g u), \\
d(v, g v), 0,0,2 d(v, g v)
\end{array}\right) \leq 0 .
$$

Hence, by $\left(\psi_{3}\right)$, we have $d(u, g u)=d(v, g v)=0$. Thus

$$
u=g u \in G(u, v) \text { and } v=g v \in G(v, u) .
$$

Therefore $(u, v)$ is a common coupled fixed point of $F, G, f, g$. The proof is similar when $g(X)$ is assumed to be a closed subset of $X$.

Put $f=g$ in Theorem 7 , we get the following result:

Corollary 8. Let $(X, d)$ be a complete metric space. Assume $F, G: X \times$ $X \rightarrow C B(X)$ and $g: X \rightarrow X$ be mappings satisfying (2.1), (2.2), (2.3) and

(8.1) $\{F, g\}$ and $\{G, g\}$ are $w$-compatible.

Then $F, G, g$ have a common coupled fixed point.

Put $F=G$ and $f=g$ in Theorem 7 , we get the following result: 
Corollary 9. Let $(X, d)$ be a complete metric space. Assume $F: X \times X \rightarrow$ $C B(X)$ and $g: X \rightarrow X$ be mappings satisfying (2.3), (3.1), (3.2) and

(9.1) $\{F, g\}$ is w-compatible.

Then $F$ and $g$ have a common coupled fixed point.

Examples 1-3 and Theorem 7 imply the following:

Corollary 10. Let $(X, d)$ be a complete metric space. Assume $F, G$ : $X \times X \rightarrow C B(X)$ and $f, g: X \rightarrow X$ be mappings satisfying (1.1), (4.1), and (7.1) and (7.2), then $F, G, f, g$ have a common coupled fixed point.

Examples 1-3 and Corollary 9 imply the following:

Corollary 11. Let $(X, d)$ be a complete metric space. Assume $F, G$ : $X \times X \rightarrow C B(X)$ and $g: X \rightarrow X$ be mappings satisfying (2.1), (2.3), (5.1) and (8.1), then $F, G, g$ have a common coupled fixed point.

Examples 1-3 and Corollary 9 imply the following:

Corollary 12. Let $(X, d)$ be a complete metric space. Assume $F: X \times X \rightarrow$ $C B(X)$ and $g: X \rightarrow X$ be mappings satisfying (2.3), (3.1), (6.1) and (9.1), then $F$ and $g$ have a common coupled fixed point.

\section{REFERENCES}

[1] M. Abbas, L. Ćirić, B. Damjanović and M. A. Khan, Coupled coincidence point and common fixed point theorems for hybrid pair of mappings, Fixed Point Theory Appl., 2012, 2012:4.

[2] M. Abbas and B. E. Rhoades, Common fixed point theorems for hybrid pairs of occasionally weakly compatible mappings satisfying generalized contractive condition of integral type, Fixed Point Theory Appl., Volume 2007, Article ID 54101, 9 pages.

[3] R. P. Agarwal, Z. Kadelburg and S. Radenović, On coupled fixed point results in a symmetric G-metric spaces, J. Inequal. Appl., 2013, 2013:528.

[4] A. Aliouche and V. Popa, General common fixed point theorems for occasionally weakly compatible hybrid mappings and applications, Novi Sad J. Math., 39 (1) (2009), 89-109.

[5] I. Altun and H. Simsek, Some fixed point theorems on ordered metric spaces and application, Fixed Point Theory Appl., Volume 2010, Article ID 621469, 17 pages.

[6] V. Berinde, Coupled fixed point theorems for $\varphi$-contractive mixed monotone mappings in partially ordered metric spaces, Nonlinear Anal., 75 (2012), 3218-3228.

[7] V. Berinde and F. Vetro, Common fixed points of mappings satisfying implicit contractive conditions, Fixed Point Theory Appl., 2012, 2012:105.

[8] T. G. Bhaskar and V. Lakshmikantham, Fixed point theorems in partially ordered metric spaces and applications, Nonlinear Anal., 65 (7) (2006), 1379-1393.

[9] H. Bouhadjera and V. Popa, Some common fixed point theorems for occasionally weakly compatible mappings satisfying implicit relation and contractive modulus, Fasc. Math., 51 (2013).

[10] L. Ćirić, B. Damjanović, M. Jleli and B. Samet, Coupled fixed point theorems for generalized Mizoguchi-Takahashi contractions with applications, Fixed Point Theory Appl., 2012, 2012:51. 
[11] B. Deshpande and R. Pathak, Fixed point theorems for noncompatible discontinuous hybrid pairs of mappings on 2-metric spaces, Demonstr. Mathe., XLV (1) (2012), 143-154.

[12] B. Deshpande and S. Chauhan, Common fixed point theorems for hybrid pairs of mappings with some weaker conditions in 2-metric spaces, Fasc. Math., 46 (2011), $37-55$.

[13] B. Deshpande and S. Chauhan, Fixed points for two hybrid pairs of mappings satisfying some weaker conditions on noncomplete metric spaces, Southeast Asian Bull. Math., 35 (2011), 851-858.

[14] B. Deshpande and A. Handa, Nonlinear mixed monotone-generalized contractions on partially ordered modified intuitionistic fuzzy metric spaces with application to integral equations, Afr. Mat., DOI 10.1007/s13370-013-0204-0.

[15] B. Deshpande, S. Sharma and A. Handa, Tripled fixed point theorem for hybrid pair of mappings under generalized nonlinear contraction, J. Korean Soc. Math. Educ. Ser. B: Pure Appl. Math., 21 (1) (2014), 23-38.

[16] H. S. Ding, L. Li and S. Radenović, Coupled coincidence point theorems for generalized nonlinear contraction in partially ordered metric spaces, Fixed Point Theory Appl., 2012, 2012:96.

[17] Z. Golubović, Z. Kadelburg and S. Radenović, Coupled coincidence points of mappings in ordered partial metric spaces, Abstr. Appl. Anal., 2012, Article ID 192581, 18 pages.

[18] M. Jain, K. Tas, S. Kumar and N. Gupta, Coupled common fixed point results involving $a \varphi-\psi$ contractive condition for mixed $g$-monotone operators in partially ordered metric spaces, J. Inequal. Appl., 2012, 2012:285.

[19] I. Kubiaczyk and B. Deshpande, Coincidence point for noncompatible multivalued mappings satisfying an implicit relation, Demonstr. Math. XXXIX (4) (2006), 555562.

[20] V. Lakshmikantham and L. Ćirić, Coupled fixed point theorems for nonlinear contractions in partially ordered metric spaces, Nonlinear Anal., 70 (12) (2009), 4341-4349.

[21] W. Long, S. Shukla and S. Radenović, Some coupled coincidence and common fixed point results for hybrid pair of mappings in 0-complete partial metric spaces, Fixed Point Theory Appl., 2013, 2013:145.

[22] Y. Liu, J. Wu and Z. Li, Common fixed points of single-valued and multivalued mappings, Int. J. Math. Math. Sci., 2005:19 (2005), 3045-3055.

[23] N. V. Luong and N. X. Thuan, Coupled fixed points in partially ordered metric spaces and application, Nonlinear Anal., 74 (2011), 983-992.

[24] H. K. Nashine, Common fixed point theorems under implicit relations on ordered metric spaces and application to integral equations, Bull. Math. Sci., 3 (2013), 183204.

[25] V. Parvaneh, J. R. Roshan and S. Radenović, Existence of tripled coincidence point in ordered b-metric spaces and applications to a system of integral equations, Fixed Point Theory Appl., 2013, 2013:130.

[26] V. Popa, A general fixed point theorem for weakly compatible mappings in compact metric spaces, Turk. J. Math., 25 (2001), 465-474.

[27] V. Popa and A. M. Patriciu, A general fixed point theorem for mappings satisfying an $\varphi$-implicit relation in complete $G$-metric spaces, Gazi University Journal of Science, 25 (2) (2012), 403-408.

[28] K. P. R. Rao, G. Ravi Babu and V. C. C. Raju, A common fixed point theorem for two pairs of occasionally weakly semi-compatible hybrid mappings under an implicit relation, Math. Sci., 1 (3) (2007), 01-06. 
[29] K. P. R. Rao and K. R. K. Rao, A common fixed point theorem for two hybrid pairs of mappings in b-metric spaces, Int. J. Anal., Volume 2013, Article ID 404838, 4 pages.

[30] S. Radenović, A note on tripled coincidence and tripled common fixed point theorems in partially ordered metric spaces, Appl. Math. Comput., 236 (2014), 367-372.

[31] B. Samet, Fixed point results for implicit contractions on spaces with two metric, J. Inequal. Appl., 2014, 2014:84.

[32] B. Samet, E. Karapinar, H. Aydi and V. C. Rajić, Discussion on some coupled fixed point theorems, Fixed Point Theory Appl., 2013, 2013:50.

[33] S. Sedghi, I. Altun and N. Shobe, A fixed point theorem for multi-mappings satisfying an implicit relation on metric spaces, Appl. Anal. Discrete Math., 2 (2008), 189-196.

[34] S. Sharma and B. Deshpande, Compatible multivalued mappings satisfying an implicit relation, Southeast Asian Bull. Math., 30 (2006), 535-540.

[35] S. Sharma and B. Deshpande, On compatible mappings satisfying an implicit relation in common fixed point consideration, Tamkang J. Math., 33 (3) (2002), 245-252.

[36] S. Sharma, B. Deshpande and R. Pathak, Common fixed point theorems for hybrid pairs of mappings with some weaker conditions, Fasc. Math., 39 (2008), 71-84.

[37] W. Sintunavarat, P. Kumam and Y. J. Cho, Coupled fixed point theorems for nonlinear contractions without mixed monotone property, Fixed Point Theory Appl., 2012, 2012:170.

[38] D. Turkoglu and I. Altun, Fixed point theorem for multivalued mappings satisfying an implicit relation, Tamkang J. Math., 39 (3) (2008), 247-253.

(Received: June 26, 2014)

(Revised: December 30, 2014)
Department of Mathematics Govt. P. G. Arts \& Science College Ratlam (M. P.) India

bhavnadeshpande@yahoo.com

amrishhanda83@gmail.com 